Revista Digital Año 6. $N^{\circ} 7$ - Año 2015. --pág. 1-81

ISSN 1853-1393

Resistencia. Chaco. Argentina - 2015

\title{
CONOCIMIENTO DIDÁCTICO DEL CONTENIDO Y MODELO DIDÁCTICO EN CIENCIAS NATURALES. EL CASO DE PROFESORAS PRINCIPIANTES DE EDUCACIÓN INICIAL
}

\section{PEDAGOGICAL CONTENT KNOWLEDGE AND DIDACTIC MODEL IN NATURAL SCIENCES. THE CASE OF KINDERGARTEN NOVEL TEACHERS.}

Mg. Margarita C. Ortiz ${ }^{1}$ - Prof. Zulema del Carmen Nussbaum ${ }^{2}$-Prof. Johanna L. Cochia ${ }^{3}$

Fecha de recepción: 24-09-2015

Fecha de aceptación y versión final: 29-10-2015

Resumen: Presentamos los resultados preliminares de un estudio realizado en el marco del proyecto "La construcción del Conocimiento Didáctico del Contenido (CDC) de profesores experimentados y principiantes de la Universidad Nacional del Nordeste. Estudio de casos múltiples".

Para esta comunicación analizamos las dimensiones de este constructo a través de la estrategia metodológica del estudio de casos (Stake, 2007; Neiman y Quaranta, 2006; Martínez Sánchez,2000).

Entendemos que el Conocimiento Profesional Docente (CPD) tiene varios componentes, ente los cuales, Reynolds (1992) incluye al Conocimiento Didáctico del Contenido.

Las investigaciones sobre el Conocimiento Didáctico del Contenido (Marcelo, 2006) refieren a estudios en los que se analiza específicamente el conocimiento que los profesores poseen respecto del contenido disciplinar, al modo en que lo transforman en conocimiento enseñable (Shulman, 1987) para hacerlo comprensible a los estudiantes y a su reconstrucción mediada por procesos de análisis y reflexión en torno de lo actuado (Schön, 1983).

Concebimos al Conocimiento Didáctico del Contenido como marco que contribuye a comprender el papel de los diferentes componentes del modelo didáctico y sus vínculos al analizar las prácticas de las profesoras.

Para este estudio, utilizamos el modelo didáctico propuesto por Estany \& Izquierdo (2001) constituido por fundamentos epistemológicos, psicológicos, socio-pedagógicos y principios didácticos y elementos, a saber: finalidad, contenidos, comunicación y organización.

1 Profesora Adjunta de Metodología de la Investigación- Licenciatura en Educación Inicial. Magister en Epistemología y Metodología de la Investigación Científica. Co-dirige el Proyecto "La construcción del conocimiento Didáctico del Contenido (CDC) de profesores experimentados y principiantes de la Universidad Nacional del Nordeste. Estudio de casos múltiples", con sede en el Instituto de Investigaciones en Educación (IIE) del Departamento de Ciencias de la Educación-Facultad de Humanidades (UNNE). Correo electrónico: mortizgaleano11@gmail.com

${ }^{2}$ Profesora de Educación Inicial. Adjunta de Administración Escolar y Auxiliar Docente de los Talleres I y II-Profesorado de Educación Inicial. Integra el equipo del Proyecto "La construcción del conocimiento Didáctico del Contenido (CDC) de profesores experimentados y principiantes de la Universidad Nacional del Nordeste. Estudio de casos múltiples", con sede en el Instituto de Investigaciones en Educación (IIE) del Departamento de Ciencias de la Educación-Facultad de Humanidades. Correo electrónico: zulema cn@hotmail.com

${ }^{3}$ Profesora de Ciencias de la Educación. Técnico-docente del Programa UNNE-Virtual del Rectorado de la UNNE. Tesista e integrante del equipo del Proyecto "La construcción del conocimiento Didáctico del Contenido (CDC) de profesores experimentados y principiantes de la Universidad Nacional del Nordeste. Estudio de casos múltiples", con sede en el Instituto de Investigaciones en Educación (IIE) del Departamento de Ciencias de la Educación-Facultad de Humanidades. Correo electrónico: johannacochia@gmail.com. 
A fin de obtener información a efectos de explorar los modelos didácticos y su relación con las dimensiones del CDC, seleccionamos cuatro profesoras principiantes de Educación Inicial, que acreditan un año de antigüedad.

Dado que los docentes noveles deben enseñar y aprender a enseñar, adquirir un adecuado conocimiento y competencia profesional en escaso tiempo, el del tránsito de estudiantes a profesores (Feiman, 2001 b; Marcelo,1999 a; Esteve,1997; Veenman,1984); optamos por trabajar- en esta primera instancia-, con el formato de guión conjetural (Bombini, 2006; Bombini y Labeur,2013) como una de las formas de abordar la planificación, basada en la narrativa y el conocimiento práctico del docente.

La narrativa implica una experiencia cognitiva de anticipación de la enseñanza y desde este enfoque, la planificación da cuenta de la dimensión subjetiva del docente, quien pone en acción sus saberes prácticos. En consecuencia, escribir la planificación es un acto cognitivo, una "construcción metodológica" (Edelstein y Coria 2005) que articula los procesos reflexivos en torno a qué y cómo se enseña y qué y cómo se aprende.

Aunque los resultados están en proceso de construcción, respecto del modelo didáctico y el CDC encontramos ciertas coincidencias con los hallazgos de Kaufman (1999), Marcelo (1998) y Jiménez Narváez (2006).

Palabras clave: docentes principiantes; conocimiento didáctico del contenido; modelo didáctico; inducción en la enseñanza.

Summary: This article presents the preliminary results of a research study carried out as part of the research Project "Pedagogical Content Knowledge construction in experienced and novel professors at Northeast National University. Multiple case study".

In this report we analize the dimensions of this construct by means of the methodological strategy of case studies (Stake, 2007; Neiman y Quaranta, 2006; Martínez Sánchez,2000).

It is understood that Professors' Professional Knowledge (PPK or CPD in Spanish) consists of various components; one of them is Pedagogical Content Knowledge (PCK) according to Reynolds (1992).

Research on Pedagogical Content Knowledge (Marcelo, 2006) refer to studies in which the specific object of analysis professors' knowledge of the disciplinary content and how this knowledge is turned into teachable knowledge (Shulman, 1987) to make it comprehensible for the students, and how this is reconstructed by means of analysis and reflection processes on the professors' practice (Schön, 1983).

We think of Pedagogical Content Knowledge as a frame that contributes to understanding the role of the different components of the didactic model and its links when analyzing the professors' practices.

For this study, we used the didactic model proposed by Estany \& Izquierdo (2001), constituted by epistemological, psychological, socio-pedagogical foundations, didactic principles and elements, namely: purpose, contents, communication and organization.

In order to explore the didactic models and their relations with the dimensions of the PCK, we selected 4 Early Education beginning professors, with just one year of teaching experience.

As novel teachers have to teach and learn how to teach, develop a proper knowledge and professional competence in a short period of time, that of the transition from student to professor (Feiman, 2001 b; Marcelo,1999 a; Esteve,1997; Veenman,1984); we decided to work - in this preliminary studywith the format of conjectural script (Bombini, 2006; Bombini \& Labeur,2013) as one of the ways to approach planning, based on professors' narrative and practical knowledge.

Narrative implies a cognitive experience of anticipating teaching and from this approach, planning reveals the teacher's subjectivity as he sets into acting his practical knowledge. As a consequence, writing the plan is a cognitive act, a "methodological construction" (Edelstein y Coria 2005) that links up reflective processes around what and how it is taught and what and how it is learned.

Although the results are still in a process of construction, the study has found coincidences as regards the didactic model and PCK with Kaufman (1999), Marcelo (1998) and Jimenez Narváez' (2006) findings.

Keys words: beginning professors, pedagogical content knowledge, didactic model, induction into teaching.

\section{Introducción}

Numerosos estudios señalan que la etapa de iniciación en la enseñanza suele ser problemática para los profesores y las profesoras noveles o principiantes. 
A partir de la premisa de que una de las competencias claves en el ejercicio profesional, es la capacidad de transformar los conocimientos disciplinares en formas de conocimiento apropiadas para los estudiantes y para la tarea específica de enseñanza en cada una de las áreas curriculares; presentamos los resultados preliminares de un estudio realizado en el marco del proyecto "La construcción del conocimiento Didáctico del Contenido (CDC) de profesores experimentados y principiantes de la Universidad Nacional del Nordeste. Estudio de casos múltiples".

Entendemos por un lado, que este constructo opera como marco que contribuye a comprender el papel de los diferentes componentes del modelo didáctico y sus vínculos al analizar las prácticas de profesoras y profesores de Educación Inicial y por otro, que un punto de partida estratégico en la formación continua es el análisis de las prácticas pues permite recuperar las propias ideas, pensamientos, palabras, decires (narrativas), los que se interpretan en articulación con aspectos estructurales e institucionales. El análisis aporta elementos sustantivos para la reflexión y el desarrollo profesional en el ámbito universitario (formación inicial) e instituciones de Educación Inicial.

La indagación pretende dar cuenta de lo que implica el conocimiento didáctico del contenido de las Ciencias Naturales y cómo lo significan/construyen las profesoras noveles de Educación Inicial, cuáles características asume el modelo didáctico en el área y sus relaciones con el CDC.

Los objetivos del estudio son:

$\checkmark \quad$ Caracterizar las dimensiones del CDC de Ciencias Naturales de las profesoras principiantes de Educación Inicial seleccionadas para el estudio

$\checkmark \quad$ Caracterizar el modelo didáctico en la enseñanza del área de cada profesora participante.

$\checkmark \quad$ Determinar relaciones entre el modelo didáctico de enseñanza de las Ciencias Naturales y el constructo CDC.

\section{Referentes teóricos}

Tres categorías teóricas orientan el análisis e interpretación de la información empírica: Conocimiento Didáctico del Contenido; modelo didáctico y docente principiante o novel.

\subsection{Conocimiento Didáctico del Contenido}

Entendemos que el Conocimiento Profesional Docente (CPD) tiene varios componentes, entre los cuales, Reynolds (1992) incluye al Conocimiento Didáctico del Contenido (CDC) o PCK (Pedagogical Content Knowledge) en términos de Shulman (1986). Usamos indistintamente CDC y PCK.

El PCK es un constructo multidimensional que describe la capacidad de un docente para transformar pedagógicamente el conocimiento del contenido disciplinar que posee, en formas y estructuras comprensibles para los estudiantes (Shulman, 2005; Bolívar, 2005; Grossman, 2005; Marcelo, 1992).

Las investigaciones sobre el Conocimiento Didáctico del Contenido (Marcelo, 2006) refieren a estudios en los que se analiza este proceso de transformación y su reconstrucción mediada por el análisis y la reflexión (Schön, 1998).

La investigación de este constructo en los futuros profesores de ciencias cobró significado desde el planteamiento que realizara Shulman al rotularlo como el paradigma perdido en las investigaciones sobre la enseñanza.

EI CDC se construye a partir del conocimiento del contenido que los profesores y las profesoras poseen, del conocimiento pedagógico general, del conocimiento de 
los estudiantes (Gudmundsdottir y Shulman, 1987) y de la propia biografía personal y profesional de las profesoras y los profesores (Butt, Raymond y Yamagishi, 1988).

Se trata de un conocimiento profesional que construyen y reconstruyen los/as docentes durante las etapas de la formación inicial y continua y se caracteriza por ser dinámico (evoluciona) e implicar la transformación de otros tipos de conocimiento (Abell, 2008).

Asumimos la propuesta de Magnusson et al (1999)

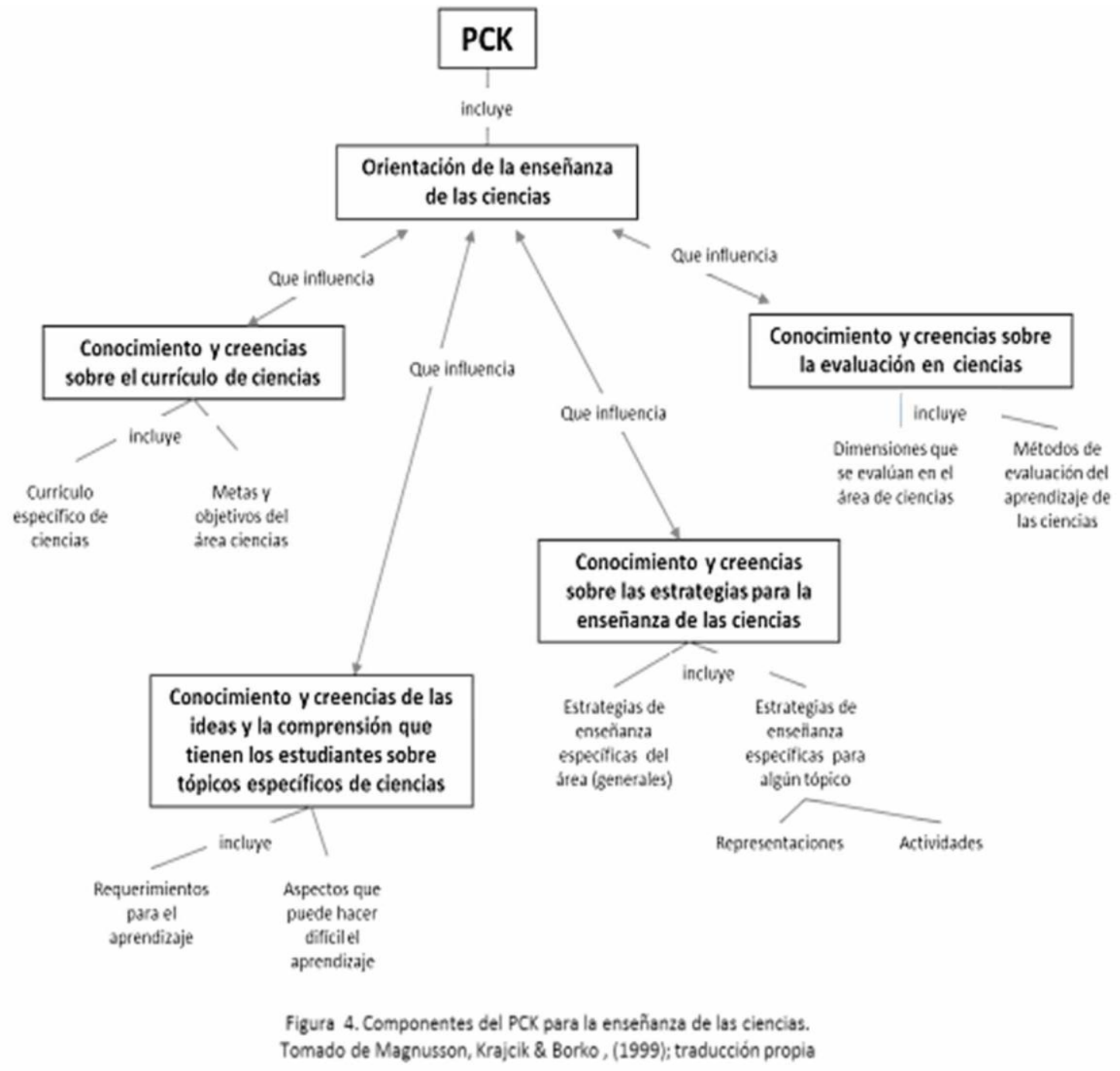

Un componente central es el conocimiento disciplinar, ya que su dominio incide en la construcción del modelo didáctico. Cuando dicho conocimiento disciplinar corresponde a profesores y profesoras de Biología, Física y Química, éstos enfatizan conceptos y teorías, la resolución de problemas y la investigación; mientras que aquellos que se desempeñan en los niveles inicial y primario y sólo han tenido una o dos asignaturas de ciencias, al enseñar acentúan procedimientos y hechos (Putman y Borko,2000).

No obstante, Kauffman, en sus investigaciones sobre el modelo didáctico de docentes de Educación Inicial encontró que no es lo mismo lo que un docente enseña que lo que sabe, es decir, que existe una significativa distancia entre que lo que enseña y el saber disciplinar, por lo que pareciera que el conocimiento disciplinar no 
es el principal determinante en este proceso en la Educación Inicial (Kaufman, 1999:100).

\subsection{Modelo Didáctico para la enseñanza de las Ciencias Naturales}

Las pocas indagaciones en torno a la enseñanza de las ciencias en la Educación Inicial develan escaso conocimiento científico por parte de profesores y profesoras (Karellry y Psillos, 2001; Garbett, 2003; Jiménez Narváez, 2006).

El Grupo de Educación en Ciencias Experimentales y Matemática (GECEM) de Colombia encontró relaciones entre el modelo didáctico y el CDC, las que se descriptas por Jiménez Narváez en su tesis doctoral con profesores de Educación Primaria.

Sobre la base de este estudio, se toman los componentes del modelo didáctico de Estany e Izquierdo (2001):

-Fundamentación epistemológica: refiere a las concepciones, creencias sobre la naturaleza de la ciencia, sus procesos de construcción, fines que persigue, estatuto de las teorías y leyes científicas y la relación ciencia-tecnología- sociedad. (CTS).

-Fundamentación psicológica: características del desarrollo, comportamiento y aprendizaje de los estudiantes.

-Fundamentación socio-pedagógica: características del contexto escolar y formas de comunicación en el aula.

-Principios didácticos: fundamentos de actuación que guían la práctica docente, esto es, decisiones referidas a las construcciones metodológicas (actividades, momentos, materiales, lugares, entre otros) y cuyos elementos son:

- para qué y por qué enseñar ciencias ( finalidad) ;

- qué enseñar (contenidos);

- características del lenguaje docente- estudiantes para compartir y construir conocimientos (comunicación) y

- recursos, actividades y secuenciación (organización),

En cuanto a las concepciones epistemológicas y didácticas de los/as docentes Porlán y Rivero (1998) sostienen que éstas tienen como eje orientador los saberes metadisciplinares vinculados con el conocimiento disciplinar y el conocimiento cotidiano.

\subsection{Profesores Principiantes o noveles}

Consideramos que los docentes somos portadores y creadores de saberes, sujetos con conocimientos y un saber hacer emerge de su propia actividad, que asume su práctica a partir de sus propios significados, estructurando y orientando su acción pedagógica (Tardif, 2004).

El docente principiante (Gimeno Sacristán, 1992; Marcelo,2007) o debutante (Cornejo Abarca,1999; Imbernón, 1994) o novato (Alliaud,2004) o novel, es quien se incorpora o inserta en el ejercicio de la docencia con un máximo de antigüedad entre 3 a 5 años.

Estos primeros desempeños en el desarrollo profesional constituyen el proceso de socialización en la práctica o inducción profesional (Imbernón, 1997) o inserción en la enseñanza (Marcelo, ibíd.) y se caracterizan por la singularidad de sus problemáticas.

Los estudios sobre profesores noveles en su inserción laboral denotan dificultades en relación con la planificación y su concreción en el aula (enseñanza), entre otros. 
En la década del '80, Veenman (1984) estudió los problemas vivenciados por estos profesores, mientras que en Latinoamérica, una referente es Jiménez Narváez (2006, 2013), por lo que coincidimos con Cornejo (1999) al señalar que es un objeto de estudio por construir, a pesar de las políticas educativas de acompañamiento a docentes niveles (INFD,2007)

De acuerdo con Marcelo $(2007,2008,1998)$ en los primeros años de enseñanza surgen dudas y tensiones, pues en un breve período de tiempo los y las principiantes deben adquirir conocimientos y competencias profesionales.

\section{Estrategia metodológica}

Para esta comunicación, analizamos las dimensiones del CDC a través de la estrategia metodológica del estudio de casos (Stake, 2007; Neiman y Quaranta,2006)

A fin de obtener información que nos permita explorar los modelos didácticos y su relación con las dimensiones del CDC, seleccionamos cuatro profesoras principiantes de Educación Inicial, que acreditan un año de antigüedad en salas de 5 años, dos de las cuales desempeñaron tareas docentes en calidad de adscriptas a una disciplina del Profesorado de Educación Inicial (Didáctica de la Educación Inicial, Taller de Integración, Investigación y Práctica III).

Dado que los docentes noveles deben enseñar y aprender a enseñar, adquirir un adecuado conocimiento y competencia profesional en escaso tiempo optamos por trabajar- en esta primera instancia-, con el formato de guión conjetural (Bombini, 2006; Bombini y Labeur, 2013) como una de las formas de abordar la planificación, basada en la narrativa y el conocimiento práctico del docente.

Solicitamos a cada docente que eligiera un contenido de uno de los ejes del diseño curricular propuestos: Los seres vivos y su entorno y Los materiales y sus interacciones.

La narrativa implica una experiencia cognitiva de anticipación de la enseñanza y desde este enfoque, la planificación da cuenta de la dimensión subjetiva del docente, quien pone en acción sus saberes prácticos. En consecuencia, escribir la planificación es un acto cognitivo, una "construcción metodológica" (Edelstein y Coria, 1995) que articula los procesos reflexivos en torno a qué y cómo se enseña y qué y cómo se aprende.

A medida que se avance en el proyecto, se prevé triangular esta información con la obtenida en entrevistas previas y posteriores a las clases y la observación de éstas, atendiendo el contexto de sus biografías personales y escolares.

\section{Resultados preliminares}

Presentamos los resultados obtenidos del análisis e interpretación de las narrativas del guión conjetural. Transcribimos fragmentos de éstos para abordar la enseñanza de los contenidos seleccionados por cada una de las docentes principiantes

Guión - D1. Los seres vivos y su entorno. Contenido: El agua y el suelo dan vida.

"Los niños de esta edad tienen ideas acerca de su mundo inmediato y éstas tienen que progresar desde el jardín y durante toda la escolaridad. Como aprendí en Ciencias Naturales, voy a aplicar el método científico para que los niños conozcan las características del agua y su contaminación.

Les voy a proponer la realización de experiencias, porque aprenden observando y las experiencias les interesan. 
Vanos a realizar la experiencia 1 con el objetivo de que el niño reconozca las características del agua potable. Para ello, pediré a los niños que observen los recipientes con agua y les preguntaré: ¿Tiene color? Luego les pediré que la prueben y preguntaré ¿Tiene sabor? Y finalmente, que la huelan ¿Tiene olor?

De este modo van a aprender que el agua potable es transparente, tiene sabor agradable y no tiene olor. Como sabemos, los niños aprenderán manipulando, observando y comentando entre ellos.

Para que los niños comprendan que el agua debe tener determinadas características para ser útil a los seres vivos, voy a realizar dos experiencias.

Experiencia A: Colocaré agua potable en tres recipientes (rojo, blanco y azul) y les pediré que prueben el agua, Les preguntaré ¿son iguales?

Dejaré el recipiente blanco con agua potable, al recipiente azul le agregaré aceite y al recipiente rojo, jabón hasta que haga espuma. Les diré que observen y preguntaré ¿Dónde está más limpia el agua? ¿Cuál tomarías? ¿Por qué?

Experiencia B: Pediré a los niños que rotulen 3 macetas con plantas de alpiste con diferentes colores o dibujos

Después pediré a uno de los niños que riegue una con agua potable; a otro niño que riegue la segunda maceta con el agua aceitosa y a un tercero, que riegue la tercera con agua jabonosa.

Regarán cada dos días de igual modo.

Les preguntaré ¿Qué observan? ¿Están las tres plantitas iguales?¿Cuál es mantiene mejor? ¿Con cuál de estas aguas debemos regar las plantas?

En la siguiente clase, observaremos las características del suelo y haremos experiencias sobre la contaminación de éste. Luego daríamos el aire y haríamos experiencias para ver sus características y la contaminación"

La secuencia didáctica de la profesora (D1) refleja la versión escolarizada del método científico, la perspectiva empiro-inductivista. Los conceptos en juego son: agua potable, contaminación de agua y suelo y sus efectos en los seres vivos. agua y suelo

Guión - D2. Los seres vivos y su entorno. Contenido: Contaminación del

"A los niños les interesa la naturaleza y especialmente, la ecología. Por eso me parece importante, dar temas de ecología en el jardín para que empiecen a formar actitudes positivas, de protección de los recursos naturales.

En Naturales, con la profe dimos mucho sobre Ecología, por eso elegí el tema de la contaminación y pienso desarrollar mi clase planteando problemas para que den sus hipótesis y luego realizar las experiencias para comprobarlas. La pregunta problema que les daré es ¿¿Cómo sabemos que el agua está contaminada? ¿Qué cosas la contaminan? Los chicos darán sus hipótesis, por ejemplo, que las cosas que tira la gente al agua la contaminan, la vuelve sucia.

Les voy a mostrar materiales: 4 vasos con agua potable ( de la canilla), témperas de colores (azul y rojo), trocitos de telgopor y les propondré mantener uno de los vasos como está y qué harían para contaminar el agua de los restantes vasos con esos materiales. Luego les preguntaría: ¿Cuál es el agua que tomarías? ¿Por qué? ¿Cuál le darías a tu perrito? ¿Por qué? ¿En cuál de las aguas colocarías un pececito? ¿Por qué?

Para ver la contaminación del suelo les preguntaría ¿Es importante que para que las plantas crezcan, mantengamos la tierra limpia, sin basuras? ¿Por qué? Ellos darán sus hipótesis y para comprobarlas, haremos una experiencia

Rotulamos tres macetas con plantas de alpiste:1-2 y 3 .

En la maceta 1 echamos aceite y mojamos la tierra en forma pareja; en la 2, colocamos sobre la tierra trocitos de telgopor, de bolsitas de polietileno, tapitas cubriendo todo con basura y a la maceta 3 la dejamos como está,

Dejamos las tres plantas en las mismas condiciones de luz, calor y aire y la regamos cada 2 días durante dos semanas más o menos. 
Les preguntaré: ¿Las tres plantitas se ven iguales? ¿Cuál está en mejor estado? ¿Cuáles no? ¿Por qué? Con esta experiencia aprenden que la basura contamina el suelo y la planta necesita suelo limpio. .. Este tema de la contaminación es importante y los motiva a los chicos, son pequeños y hay que bajar a su nivel para enseñar ciencia...por eso, si bien esto es lo que planificaría, no voy a dejar de lado, los intereses de los chicos, como por ejemplo, la contaminación del agua de una laguna cercana al jardín o del rio Negro y los basurales que contaminan el suelo y aire de Resistencia y la basura en el jardín "

La versión falsacionista o hipotético -deductiva de la metodología científica aparece en la secuencia didáctica de la D2. Los contenidos de enseñanza son: contaminación de agua y suelo, efectos de la contaminación en plantas y animales, el papel del hombre en la contaminación.

Guión -D3. Los seres vivos y su entorno. Contenido: El nacimiento de una planta

"A los chicos les gusta manipular las cosas, interactuar con los objetos y son curiosos, les gusta hacer experiencias, eso es lo que más les interesa cuando se da ciencias naturales. Un tema complejo es el de los seres vivos, pero las plantas y los animales son cercanos a los niños por lo que es importante que empiecen a preguntarse sobre ellos.

Para comenzar la clase les preguntaré ¿cómo puedo obtener nuevas plantas para mi huerta o jardín? Según sus experiencias previas pueden decir, de semillas o de gajitos porque vieron a sus mamás, por ejemplo, hacer plantas de croto a partir de gajos.

Les voy a proponer hacer un germinador con semilla de porotos (G1) previamente remojadas durante 24 horas.

En un vaso trasparente colocaremos papel secante y entre éste el vidrio del vaso, las semillas de poroto separadas para poder observarlas. En el interior, colocaremos algodón y regaremos con un gotero diariamente. ¿Por qué es importante mantener la humedad? Darán sus hipótesis. ¿Qué pasará si no ponemos agua? Darán anticipaciones.

Haremos otro germinador igual (G2) al que no regaremos y un tercer germinador (G3) en una capa de Petri, donde colocaremos semillas de lentejas en agua y observaremos. ¿Qué pasará con las semillas de lentejas que pusimos en agua? Darán sus anticipaciones.

Guiaré la observación diaria de los germinadores (G1 y G3) con preguntas ¿son todas las semillas del mismo tamaño? ¿Qué sucede con las cascaritas que las recubre? ¿Aparece una colita? ¿Dónde? Y ahora ¿qué está apareciendo en la parte de arriba de la semilla? ¿Son hojitas? ¿Qué pasó con las semillas del segundo germinador'? ¿Por qué?

poroto.

Vamos a concluir que las plantas de poroto y de lenteja nacen de una semilla de

Les enseñaré la canción

Yo soy un gordo porotito

Y yo la regadera que lo riega

Yo crezco con colita y hojas

Yo ya soy una planta entera

Si bien la idea es que entiendan que las plantas nacen de una semilla, también si a los chicos les interesa, haremos plantas de gajos de croto, de incienso,etc, para ver cómo de un gajo se multiplican las plantas.

En las siguientes clases observaríamos el crecimiento de las plantas de poroto y de alpiste. Para eso, primero les voy a proponer trasplantarlas en macetas con arena y otras con tierra negra. Para medir la altura, por ejemplo, una vez por semana, utilizaría tiras de papel y las pegaríamos sobre una línea para armar un gráfico de barras. También observaríamos en cuál suelo crece mejor /arena- tierra negra)"

La D3 al igual que la D2 problematiza la realidad y adopta la versión popperiana del método científico. Contempla el interés en la secuenciación de 
actividades de enseñanza y aprendizaje. Propone abordar los contenidos germinación y multiplicación vegetativa.

Guión -D4. Los materiales y sus interacciones. Contenido: Magnetismo. El imán

"Para mi los chicos aprenden al estar en contacto con los objetos y al observarlos van aprendiendo. Para que observen trabajaré con el método científico como aprendimos en Naturales en la facu... a ellos les gusta, les interesa trabajar como pequeños científicos.

Preguntaré a los niños ¿conocen los imanes? ¿Qué artefactos de nuestras casas tienen imanes? Por ejemplo ¿por qué hay imanes en los burletes de la heladera? Así voy a relacionar Tecnología (artefactos) con la Física y partiré de conocimientos cotidianos.

¿Qué cosas son atraídas por un imán? Darán sus hipótesis y las pondremos a prueba con esta experiencia:

En una palangana o caja con virutas de madera jugaremos a ocultar y sacar objetos escondidos con un imán. Ocultaremos objetos de metal como chapitas, ganchitos, alfileres y de otros materiales: tapitas de botellas, trocitos de bolsitas de polietileno, bolitas de madera y de vidrio.

Como el juego es un recurso didáctico, jugaremos a pasar el imán por sobre las cosas escondidas. Preguntaré ¿A cuáles atrae el imán? ¿A cuáles no atrae el imán?

Comentaré que mediante la fuerza magnética pudimos separar los objetos que son atraídos por el imán de los que no lo son.

Para que puedan relacionar la fuerza magnética con la flotación-tema que ya conoceny descubran que la fuerza magnética puede actuar a través del líquido, les voy a proponer armar un pulpito con un corcho y ocho clavos.

Nuevamente jugaremos, ahora con el pulpito. Pondremos el pulpito armado en un recipiente con agua. Colocaremos un imán por fuera haciendo que el corcho suba, baje y se mueva en distintas posiciones.

Sacaremos el imán y les preguntaré: ¿Flota el corcho? ¿Queda sumergido? ¿Qué debemos hacer para que el corcho se hunda?

Quitaremos los clavos, acercaremos el imán de nuevo y preguntaré ¿Flota ahora el corchito? ¿Se mueve hacia el imán? ¿Qué sucede?

Esto es lo que yo voy a enseñar, pero también tendré en cuenta otras cosas que surjan según los intereses de los chicos"

Este guión también sustenta la versión falsacionista del método científico. Propone trabajar los conceptos físicos de fuerza magnética y flotación.

En los guiones conjeturales de las cuatro docentes, las actividades ocupan un lugar relevante.

Expresiones como "los niños aprenderán manipulando, observando y comentando entre ellos" (D1)... "al estar en contacto con los objetos y al observarlos van aprendiendo" (D4)... dan cuenta de que la construcción de conceptos deviene de la manipulación de los objetos.

También, al hacer referencia a la simplificación de los conceptos para enseñarlos, "son pequeños y hay que bajar a su nivel" (D2) revelan una concepción de aprendizaje factual (Hollon y Anderson, 1987, citados en Porlán y Rivero, íbid).

El poner el énfasis en los estudiantes como centro del currículo, para que éstos se expresen, participen y aprendan en un clima natural, en el que sus intereses operan como uno de los elementos organizadores, denota el enfoque espontaneísta en sus modelos didácticos.

Tres de las docentes propician la indagación acerca de las necesidades de los seres vivos para su desarrollo, focalizando el agua y el suelo como recursos naturales valiosos que hay que cuidar de modo responsable individual y colectivamente. 
En relación con el conocimiento y creencias sobre el currículum de ciencias, su enseñanza y aprendizaje ( fundamentos epistemológicos), observamos una tendencia empiro-inductivista y falsacionista de las Ciencias Naturales (Porlán y Riveros, 1998; Ortiz, 2002).

Una de las profesoras (D1) manifiesta un pensamiento empirista radical, basado en la creencia de que la observación permite obtener por inducción un conocimiento objetivo y verdadero de la realidad (objetivismo, absolutismo y realismo); mientras que las restantes, dan cuenta de un empirismo moderado, es decir cercano al falsacionismo popperiano en el que la hipótesis y la experimentación sustituyen la observación como eje fundamental del proceso científico al hacer ciencia escolar. Se trata de un inductivismo matizado o falsacionismo experimentalista (Porlán,1989).

Sin embargo, la mayor representatividad de la concepción empirista de la ciencia, no se traduce en los modos de entender los procesos de enseñanza y aprendizaje.

Las docentes conciben al contenido del conocimiento escolar como una adaptación del conocimiento disciplinar (tendencia curricular tecnológica) y con algunos rasgos del del contenido como adaptación contextual del conocimiento cotidiano (enfoque curricular espontaneísta).

En lo metodológico, los objetivos son el hilo conductor de las actividades en la versión fuerte del empirismo o bien, lo son los intereses de los estudiantes (versión débil del empirismo).

Esta epistemología escolar es consistente con la imagen empirista de la ciencia y el modelo didáctico personal (tecnológico-espontaneísta)

Asimismo, en relación con la fundamentación psicológica, los relatos incluyen imágenes de ideas próximas al aprendizaje por asimilación de significados. Por ello, la metodología de enseñanza se basa en secuencias cerradas de actividades y en dos casos, en secuencias también orientadas por los intereses de niños y niñas (D2 y D4).

En cuanto a los fundamentos socio-pedagógicos, las profesoras principiante señalan que en el contexto escolar las Ciencias Naturales tienen un rol secundario y que las formas de comunicación en el aula se basan en el diálogo docente-alumnos/as y ocasionalmente, dialogo en pequeños grupos.

Las decisiones referidas a las construcciones metodológicas ponen el énfasis en los elementos que hacen a la organización: recursos, actividades y secuenciación

Los decires dejan ver algunas dificultades que enfrentan las profesoras principiantes: la gestión de la clase (Veenman, 1984; Marcelo, 1992) y la planificación de secuencias didácticas, debido a la elevada complejidad cognitiva que demanda (Magnusson et al., 1999).

\section{A modo de consideraciones finales}

Los resultados han permitido la caracterización parcial del CDC y del modelo didáctico para la enseñanza de las Ciencias Naturales sostenido por las profesoras de Educación Inicial participantes en el estudio.

Respecto de los vínculos entre modelo didáctico y CDC o PCK, los datos están en construcción y aunque observamos coincidencias en algunos aspectos con el estudio de Kaufman (1999), pensamos que es necesario contar con otras fuentes, como las entrevistas y observaciones de clases. .

\section{BIBLIOGRAFİA}


Abell, S. K.(2008). Twenty years later: does pedagogical content knowledge remain a useful idea? En: International Journal of Science Education, 30 (10), 1405-1416.

Alliaud,A. (2004) "La experiencia escolar de maestros inexpertos. Biografías. Trayectorias y práctica profesional", en Revista Iberoamericana de Educación № 34 , pp.3.-14.Disponible en: http://www.rieoei.org/profesion33.html.

Bolívar, A. (1993). Conocimiento didáctico del contenido y formación del profesorado. En: Revista Interuniversitaria de Formación del Profesorado (16), 113- 124. Eneroabril.

(2005). Conocimiento didáctico del contenido y didácticas específicas. En:

Revista de currículum y formación del profesorado. Universidad de Granada: Profesorado.

Bombini, G. Y Labeur, P. Escritura en la formación docente: los géneros de la práctica. Enunciación Vol. 18, No. 1/ enero-junio de 2013 Bogotá, Colombia pp. 19 29.Disponible en:file://C:/Users/usuario/Downloads/DialnetEscrituraEnLaFormacionDocente-782069.pdf

Bombini, G. (2006). "Capítulo 4. Vidas de profesores". Reinventar la enseñanza de la lengua y la literatura. Buenos Aires, Libros Del Zorzal. pp. 101-120.

Butt, R.; Raymond, D. y Yamagishi, L. (1988). Autobiographic praxis: Studying the formation of teachers' Knowledge. Journal Curriculun Theorizing, 7(4), pp. 87-164.

Cornejo, J. (1999). Profesores que se inician en la docencia: algunas reflexiones al respecto desde América Latina. Revista Iberoamericana de Educación, No. 19, 51 100

Edelstein, G. y Coria, A. (1995): Imágenes e imaginación. Iniciación a la docencia. Bs. As.: Kapelusz

Estany, A. e Izquierdo, M. (2001). Didactología: una ciencia de diseño. Endoxa: Series filosóficas, No. 14, $13-33$

Garbett, D. (2003). Science education in early childhood teacher education: putting forward a case to enhance student teacher's confidence and competence. Research in science education. 33 (4), 467 - 481

Garritz, A., Daza, S y Lorenzo, G. (2014). Conocimiento didáctico del contenido. Una perspectiva iberoamericana. Editorial Académica Española, Saarbürcken /alemania. $p$ 524.

Gimeno Sacristán, J., "Profesionalización docente y cambio educativo", en: Alliaud, A., y Duschatzky, L. (comp.). 1992. Maestros. Formación, práctica y transformación escolar. Buenos Aires, Miño y Dávila Editores

Grossman, P. (1989). A study in contrast: Sources in Pedagogical Content Knowledge for secondary English. En: Journal of Teacher Education (40), 24-31.

(1990). The making of a teacher. Teacher knowledge and teacher education. New York: Teachers College, Columbia University.

Gudmundsdottir, S. Y Shulman, L. (1987). Pedagogical Content Knowledge in Social Studies. En: Scandinavian Journal of Educational Research, 31(2), 59-70.

INFD. Instituto Nacional de Formación Docente. Línea: Acompañamiento de Docentes Noveles. Disponible en: http://portales.educacion.gov.ar/infd/acompanamiento-adocentes-noveles/ Consulta: 15/04/20215.

Imbernón, F.(1994) La formación y el desarrollo profesional del profesorado. Hacia una nueva cultura profesional. Barcelona:Graó.

-(1997)"La formación del profesorado: ciertas confusiones y algunas evidencias", en Aula de Innovación Educativa,62;40-42.

Jimenez Narvaez. M.M. La profesora principiante de preescolar y su modelo didáctico para enseñar Ciencias Naturales: Un estudio de caso. Tesis Maestría en Educación con énfasis en Docencia en las Ciencias Experimentales. Medellín, 2006. Facultad de Educación. Universidad de Antioquía. Colombia. 
Profesor/a principiante de ciencias naturales: La configuración de su conocimiento en la inserción profesional. Tesis Doctorado en Educación. Medellín, 2013. Facultad de Educación. Universidad de Antioquía. Colombia.

Kallery, M. y Psillos, D. (2001). Pre-school teacher's content knowledge in science: their understanding of elementary science concepts and of issues raised by children's questions. International Journal of Early Years Education. Vol. 9, No. 3, 165 - 179.

Kaufman, M. (1999). Caracterización de modelos didácticos del nivel inicial. Algunas ideas que sustentan una manera de enseñar: el ciclo de vida de las plantas. P. 65 107.

Kaufman, M. Y Fumagalli, L. (Comp.) Enseñar ciencias naturales: reflexiones y propuestas didácticas. Buenos Aires: Paidós.

Magnusson, S.; Krajcik, J. Y Borko, H. (1999). Nature, sources and development of Pedagogical Content Knowledge for Science Teaching. En: J. Gess-Newsome y N. Lederman (Eds.). Examining Pedagogical Content Knowledge. The construct and its implications for Science Education (pp. 95-132). Dordrecht, Boston, London: Kluwer Academic Publishers.

Marcelo, C. (2007). Empezar con buen pie: inserción a la enseñanza para profesores principiantes. Docencia, 33,27-38.

---- (Coord), (2008) El Profesorado principiante. Inserción a la docencia Octaedro Disponible en: http://cedoc.infd.edu.ar/upload/El_profesorado_principiante.pdf

(1992). Cómo conocen los profesores la materia que enseñan. Algunas contribuciones de la Investigación sobre el Conocimiento Didáctico del Contenido. Congreso Internacional sobre Las didácticas específicas en la formación del profesorado. Santiago de Compostela. España.

Mellado, V. (1996). Concepciones prácticas de aula de profesores de ciencias, en formación inicial de primaria y secundaria. Enseñanza de las ciencias. 14 (3), 289 -302 Neiman, G; Quaranta, G. (2006) "Los estudios de caso en la investigación sociológica", en Vasilachis de Gialdino (comp.) Estrategias de investigación cualitativa. Buenos Aires: Gedisa

Ortiz, M.C. (2002) Concepciones epistemológicas de los formadores de formadores en torno a la teoría evolutiva como organizador curricular, Tesis de Maestría en Epistemología y Metodología de la Investigación Científica. Facultad de Humanidades. UNNE. Rcia. Chaco.

Porlán, R. y Rivero, A. (1998). El conocimiento de los profesores. Una propuesta formativa en el área de ciencias. Sevilla: Diada Editora. 213 p.

Putman, R. y Borko, H. (2000). El aprendizaje del profesor: implicaciones de las nuevas perspectivas de la cognición. 219 - 309. En: Biddle, B, Good, T. Y Goodson, I. La enseñanza y los profesores I. La profesión de enseñar. Barcelona: Paidós

Sanmartí, N. (1995). Proyecto docente e investigación de didáctica de las ciencias. Departamento de didáctica de la matemática i les ciences experimentals. Universitat Autónoma de Barcelona. Cap. 2. p. 39 - 95

Schön, D. (1998), El profesional reflexivo. Cómo piensan los profesionales cuando actúan, Editorial Paidós, Barcelona.

Stake, R. (2007) Investigación con estudios de caso. Madrid, Morata.

Tardif, M. (2004) Los saberes docentes y su desarrollo profesional. Madrid: Narcea Utges, G. (2003). El pensamiento de los profesores algunas reflexiones sobre el estado del arte. En: Revista TED Tecné, Episteme y Didaxis. Bogotá: Universidad Pedagógica Nacional. Número extra, 52 - 65

Veenman, S. (1984). Perceived problems of beginning teachers. Review of Educational Research, 54, (2), 143 -178. 